\title{
Coherent X-ray scattering reveals nature of dynamical transitions in nanoparticle-polymer suspensions
}

Article · July 2016

DOI: 10.1016/j.polymer.2016.07.048

CITATIONS

0

4 authors, including:

\section{Sivasurender Chandran}

University of Freiburg

14 PUBLICATIONS 47 CITATIONS

SEE PROFILE
READS

23 


\title{
Coherent X-ray scattering reveals nature of dynamical transitions in nanoparticle-polymer suspensions
}

\author{
Sivasurender Chandran ${ }^{\mathrm{a}, \mathrm{b}}$, N. Begam ${ }^{\mathrm{a}}$, M. Sprung ${ }^{\mathrm{c}}$, J.K. Basu ${ }^{\mathrm{a}, ~ *}$ \\ ${ }^{a}$ Department of Physics, Indian Institute of Science, Bangalore, 560012, India \\ ${ }^{\mathrm{b}}$ Institute of Physics, Albert-Ludwigs University of Freiburg, 79104, Freiburg, Germany

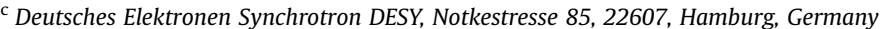

\section{A R T I C L E I N F O}

\section{Article history:}

Received 2 May 2016

Received in revised form 2 July 2016

Accepted 17 July 2016

Available online $\mathrm{xxx}$

\section{Keywords:}

Dynamics

Rheology

Suspensions

Nanoparticle-polymer mixtures

X-ray photon correlation spectroscopy

Diffusing wave spectroscopy

\begin{abstract}
A B S T R A C T
We report the microrheological behavior and dynamics of the suspensions of polystyrene-grafted gold nanoparticles (PGNPs) mixed with linear polystyrene (PS) as a function of the PS concentration and size asymmetry parameter, $\xi\left(=M_{g} / M_{m}\right.$, with $M_{g}$ and $M_{m}$ being the grafted and free chain molecular weights, respectively) $=2.76$ and 0.14 . Diffusive wave spectroscopy (DWS) is used for measuring the frequency dependent micro-rheology parameter, the loss modulus $G^{\prime \prime}(\omega)\left(\sim \omega^{\alpha}\right)$. For suspensions with $\xi=0.14$ a transition of $\alpha$, from $\sim 1$ to $\sim 1.6$, is observed at a critical fraction of added polymers. On the other hand, $\alpha$ is always $\sim 1$ for the suspensions with $\xi=2.76$. Corresponding $\mathrm{x}$-ray photon correlation spectroscopy (XPCS) data on the same samples reveals a sharp transition in the nature of dynamics of PGNPs, from exponential to logarithmic, in suspensions with $\xi=0.14$, while for suspensions with $\xi=2.76$ this dynamical transition is not observed. Interestingly, the dynamical transition in $\xi=0.14$ based samples occurs at the same fraction of added polymers, where the transition of $\alpha$ from $\sim 1$ to $\sim 1.6$ was observed, thus providing a clear correlation between microscopic dynamics and micro-rheology. The conformational transitions of the grafted polymers is found to be the reason behind the observed differences in the dynamic behavior of the two systems. The important role of advanced $\mathrm{x}$-ray scattering techniques in unravelling subtle aspects of nanoscale complex polymer and soft colloidal dynamics is thus revealed.
\end{abstract}

(c) 2016 Published by Elsevier Ltd.

\section{Introduction}

Suspensions of colloids and their mixtures with polymers have been used as model systems to understand the nature and formation of different phases like crystals, glasses and gels [1-9]. In recent times, considerable interest has been focused on the softer analogues of colloids, especially, star polymers, micelles, microgels and dendrimers [10-16]. The enhanced interest in soft colloids is largely due to their tunable interactions which leads to rich structural and dynamical behavior. For example, star polymers have shown various structures like tetrahedral, hexagonal, face centered and body centered cubic structures by just changing the functionality i.e., the number of polymers attached to the microscopic core [14]. Further, binary mixtures of star polymers have shown the presence of fascinating phases like asymmetric and double glasses and transition between these phases were achieved by simply

\footnotetext{
* Corresponding author.

E-mail address: basu@phyiscs.iisc.ernet.in (J.K. Basu).
}

changing the fraction of one of the star polymers [15,17].

Polymer grafted nanoparticles (PGNPs) forms a sub-class of the soft colloids with an inorganic core grafted with organic polymers [11,18-21]. The interest in PGNPs mainly stems from their ability to self assemble in to various structures with interesting magnetic to electric and optically properties [11,22-24]. In addition, the PGNPs when added to polymers lead to formation of a well known type of materials called the polymer nanocomposites (PNC) [11,24-28]. Thanks to the intense efforts of various researchers [25-32], a considerable understanding of the structure of PGNP-polymer mixtures have been achieved in the melt state. The phase behavior of PGNP-polymer mixtures (with identical chemical structures) can be understood through the parameter $\xi$, which is defined as the ratio of the molecular weights of grafting and matrix polymer i.e., $\xi=M_{g} / M_{m}$, where $M_{g}$ and $M_{m}$ are the grafting and matrix molecular weights, respectively [26,28]. For mixtures with $\xi>1$, stretching energy of grafting polymers are lower than the gain in the entropic contribution due to the interpenetration of grafting and matrix chains. Hence $\xi>1$ favors the wetting of grafted layer (the polymer brush) by the matrix polymers and the grafted 
polymers of the PGNPs in this condition is known as wet brush. On the other hand, for mixtures with $\xi<1$, the penalty for allowing the interpenetration of grafting and matrix polymers will be considerably higher than the entropic gain. As a consequence, $\xi<1$ promotes phase separation and the grafted polymers are called the dry brush. Even the systems with $\xi<1$, has been shown to wet the matrix polymers by varying various other parameters like grafting density of the PGNPs, polydispersity of the grafted layers $[30,33,34]$, stiffness of the grafted chains [35] and the fraction of added polymers $[26,36]$. The differences in phase behavior with different $\xi$ values were captured by the variation in the glass transition temperature and the viscosity of the mixtures [25,28]. While a large volume of data exists for PNCs, equivalent studies on PGNP-polymer binary suspensions are clearly lacking except for few recent reports $[21,37,38]$. We have recently [21] observed intriguingly similar phase behavior for systems with different $\xi$ values in the suspensions through the combination of small angle $\mathrm{x}$-ray scattering, coarse grained molecular dynamic simulations and integral equation theory. In addition, we have also observed anomalous dynamic behaviors for PGNP polymer mixtures in both melt and solutions $[19,20,37,39]$.

In the current report, we build on this premise through systematic study of structure, microrheology and dynamics of PGNPpolymer mixtures through a combination of small angle $\mathrm{x}$-ray scattering (SAXS), dynamic light scattering (DLS), x-ray photon correlation spectroscopy (XPCS) and diffusing wave spectroscopy (DWS). We have mixed polystyrene grafted gold nanoparticles with different fractions of linear polystyrene (PS) of two different molecular weights to obtain $\xi=0.14$ and 2.76. By changing the $\xi$ value of the mixtures, we have observed surprising differences in the frequency dependence of the loss modulus (DWS) and the nature of relaxations (XPCS) for the nanoparticle-polymer suspensions. A dynamical transition seems to emerge above a certain concentration of added polymers for the $\xi=0.14$ systems. Irrespective of the $\xi$ values, though, both the mixtures show a slowing down of dynamics (increase in viscosity and relaxation time), our DLS experiments have highlighted the possibly different physics behind the slowing down for both the systems. To understand these unexpected but well supported results, we have proposed a model, inspired from the conventional colloids and star polymers, which includes the differences in the conformation of the grafted polymers in the presence of linear polymers of different molecular weights. Our work indicates the intriguing physical behavior exhibited by PGNP-polymer binary suspensions at very low PGNP concentrations and the need for further experimental and theoretical investigations to understand the phase and dynamical behavior of nanoparticle-polymer suspensions.

\section{Materials and methods}

\subsection{Sample preparation and details}

The results presented here are based on the concentrated solutions (concentrations of the order of the overlap concentration, $C^{*}$ and above) of binary mixtures of polystyrene grafted gold nanoparticles (PGNPs) and linear polystyrene (PS) in toluene, a good solvent for both PGNPs and PS. PGNPs were synthesized using methods described earlier $[19-21,40]$ and characterized by various techniques viz., transmission electron microscopy (TEM), thermogravimetric analysis (TGA) and DLS. TEM image of the PGNPs is shown in Fig. 1 . The hydrodynamic radius, $R_{h}$, of PGNPs was obtained from DLS. The defining characteristics of the PGNPs are tabulated in Table 1. We have mixed two different molecular weights for the linear polystyrene with the PGNPs for obtaining two PGNP:PS size ratios, $\xi \approx 0.14$ and 2.76 (where, $\xi=M_{g} / M_{m}, M_{g}$ and

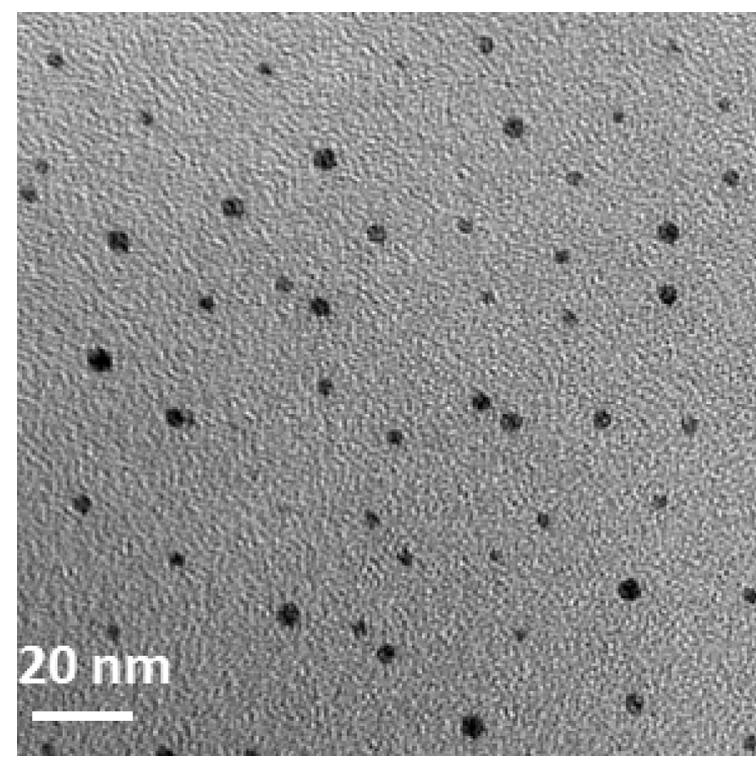

Fig. 1. A representative transmission electron micrograph of pure PGNPs is shown.

Table 1

Properties of PGNPs.

\begin{tabular}{llll}
\hline$R_{c} \mathrm{~nm}$ & $M_{g} \mathrm{~kg} / \mathrm{mol}$ & $R_{h} \mathrm{~nm}$ & $\sigma \mathrm{nm}^{-2}$ \\
\hline 1.6 & 53 & 30.0 & 1.1 \\
\hline
\end{tabular}

$R_{c}$ is the radius of the core (gold nanoparticles), $R_{h}$ is the hydrodynamic radius of the PGNP, $M_{g}$ is the molecular weight of the grafting polymer and $\sigma$ is the grafting density of the PGNPs.

$M_{m}$ being the molecular weights of grafting and matrix polymers respectively). PGNPs were dissolved in toluene by constant stirring to form homogeneous solutions. The linear polymers (polystyrene of molecular weights 19.2 and $382 \mathrm{~kg} / \mathrm{mol}$ ) were also dissolved in toluene in similar way to obtain concentrations similar to the PGNP solution. The PGNP and polymer solutions were then mixed in various proportions to obtain the samples described in Table 2. Since two solutions with similar concentrations and hence viscosities were mixed, to a first order assumption, we assume that the viscosity of all the suspensions to be similar. For all the mixtures, the polymer solutions were mixed with the PGNP solutions with the initial fraction 0.12 . This concentration indicates a mean interparticle spacing $d_{\text {calc }} \sim 30 \mathrm{~nm}$ and the ratio $d_{\text {calc }} / R_{h} \sim 1$. Accordingly, one can preempt the manifestation of the structure by the nature of interactions.

\subsection{SAXS and XPCS measurements}

SAXS and XPCS measurements were performed at P-10 beamline of PETRA-III, Deutsches Elektronen Synchrotron (DESY) using $\mathrm{X}$-ray beam of energy $8.0 \mathrm{keV}$. Since $\mathrm{X}$-rays are sensitive to the differences in electron density and due to the higher electron

Table 2

Sample details.

\begin{tabular}{lllll}
\hline$M_{m} \mathrm{~kg} / \mathrm{mol}$ & $\lambda$ & $\xi$ & $\mathrm{C}^{*} \mathrm{mg} / \mathrm{ml}$ & $\mathrm{C} / \mathrm{C}^{*}$ \\
\hline 382 & 0.95 & 0.14 & 19 & $0-12$ \\
19.2 & 5.5 & 2.76 & 180 & $0-12$ \\
\hline
\end{tabular}

$M_{m}$ is the molecular weight of the added polymers, $\lambda=R_{h} / R_{g}$ where $R_{h}$ and $R_{g}$ being the hydrodynamic radius of PGNP and polymers respectively, $C$ is the concentration of added linear chains. 
density of gold, both SAXS and XPCS are only sensitive to the gold core of PGNPs. The experiments were performed at $190 \mathrm{~K}$ to facilitate probing of the PGNP dynamics within the time window of XPCS. As PGNPs are considered athermal systems $[26,30,31]$ the temperature is not expected to play any significant role in determining the nature of the interactions. Hence, decreasing temperature would only lead to a trivial increase in the overall viscosity of the solution and hence a slower dynamics of PGNPs. For collecting SAXS and XPCS data, the suspensions were filled in capillary tubes (of diameter $\sim 1 \mathrm{~mm}$ ). A beam size of $20 \times 20 \mu \mathrm{m}^{2}$ was used for the measurements and the scattered beam was recorded on Pilatus $300 K$ detector for SAXS measurements and MAXIPIX detector for XPCS measurements as described elsewhere [41,42].

In XPCS experiments, we observe the speckle pattern (spatiotemporal intensity fluctuations) of the x-rays scattered from the sample, more specifically from the gold core of the PGNPs [19,20,37]. The temporal fluctuations will be used to measure the auto correlation function $\left(g_{2}(q, t)\right)$ and for stationary dynamics $g_{2}(q, t)$ can be related to the intermediate scattering function $(F(q, t))$ as follows

$g_{2}(q, t)=1+\beta|F(q, t)|^{2}$

where, $\beta$ is the speckle contrast, $q$ is the measured wave vector. The nature of dynamics for our suspensions have been found to be stationary by measuring two time correlation functions (not shown here) at a given wave vector $[43,44]$.

\subsection{DWS measurements}

DWS is a passive non-intrusive micro-rheological technique to measure the viscoelastic behavior of turbid suspensions. The advantages of DWS over a normal rheometer is twofold viz., (a) possibility of accessing much higher frequencies $\left(10-10^{5} \mathrm{~Hz}\right)$ and $(\mathrm{b})$ possibility of using least amount of sample $\sim 200-500 \mu \mathrm{L}$. Due to the turbidity of the sample, light experiences multiple scattering through the medium providing access to the short time dynamics of the correspondingly shorter length scales $[45,46]$. On the other hand, time scales accessible to XPCS or the frequencies accessible to normal rheometers are rather limited. Hence, DWS opens the door to probe the rheological response of suspensions at much shorter time and length scale that are not accessible to conventional rheology measurements as well as XPCS. Excellent agreements between DWS and conventional rheometers have been shown [47].

DWS measurements were done at room temperature using LS instruments, Switzerland. Argon laser with the wavelength $687 \mathrm{~nm}$ was used for the measurements. Since the PGNPs were too small to scatter the laser, we have used titania particles, with a mean radius $\sim 180 \mathrm{~nm}$, as tracers. Experiments were carried out with suspensions (mixed with Titania particles at a fraction of $1 \%$ ) filled in cuvettes (with sample volume $\sim 0.5 \mathrm{ml}$ ). The intensity autocorrelation function $\left(g_{2}(t)\right)$ as measured from DWS was further processed using the formulations devised by Mason and Weitz [46] to obtain the frequency dependence of storage and loss modulus.

In DWS experiments, the temporal fluctuations of the light scattered by the probe particles dispersed in the sample is used to measure the intensity auto-correlation function $\left(g_{2}(t)\right)$, which can then be used to measure the mean square displacement as shown below [46].

$g_{2}(t)=1+\exp \left(-\frac{1}{6} q^{2}\left\langle\Delta r^{2}(t)\right\rangle\right)$

where $q$ is the scattering vector which is related with the scattering angle $(\theta)$ by $q=4 \pi / \lambda_{d w s} \sin (\theta)$ and $\left\langle\Delta \mathrm{r}^{2}(t)\right\rangle$ is the mean square displacement (MSD) of the probe particle. Since the radius $(\sim 180 \mathrm{~nm})$ of the probe particles is larger than the size of both PGNPs and polymers, the nanoparticle-polymer suspensions will be considered a homogeneous medium with a constant viscosity. Hence, by measuring the MSD of the probe particles the microrheology of the suspensions can be measured. The MSD of the probe particles obtained from DWS is related to the complex viscoelastic modulus of the solutions using the following StokesEinstein equation [46]:

$G^{*}(\omega)=\frac{k_{B} T}{\pi a i \omega\left\langle\Delta r^{2}(i \omega)\right\rangle}=G^{\prime}(\omega)+i G^{\prime \prime}(\omega)$

where, the storage and loss modulus are respectively given by eq. (4) and eq. (5)

$G^{\prime}(\omega)=\left|G^{*}(\omega)\right| \cos (\pi b(\omega) / 2)$

and

$G^{\prime \prime}(\omega)=\left|G^{*}(\omega)\right| \sin (\pi b(\omega) / 2)$

where $a$ is the radius of the probe particle, $k_{B}$ is the Boltzmann constant, $T$ is the absolute temperature, $i$ is the imaginary number $\sqrt{-1},\left\langle\Delta \mathrm{r}^{2}(i \omega)\right\rangle$ corresponds to the Laplace transform of the MSD $\left\langle\Delta \mathrm{r}^{2}(t)\right\rangle$. $b$ approaches 1 when the probe particles move diffusively and $G^{\prime \prime}(\omega)$ dominates. For systems with higher elasticity $b$ approaches zero and $G^{\prime}(\omega)$ dominates. Using $G^{\prime \prime}(\omega)$, the viscosity of the solution can be obtained [48].

$\eta=G^{\prime \prime}(\omega) / \omega$

\subsection{DLS measurements}

We have used DLS for measuring the hydrodynamic radius $\left(R_{h}\right)$ of PGNPs in different mixtures. DLS measures the Brownian dynamics of PGNPs by measuring the intensity correlation function (ICF) [49]. The ICF was fitted with exponential function of the form shown below

$C(t)=A e^{-2 \Gamma t}+1$

where $A$ is a constant evaluated by fitting the data. $\Gamma$ is given by

$\Gamma=q^{2} D$

Here $q$ and $D$ are wave vector and diffusion coefficient, respectively. The wave vector $q$ is defined as

$q=\frac{4 \pi n}{\lambda_{d l s}} \sin (\theta)$

Where $\mathrm{n}$ is the refractive index of the medium, $\lambda_{d l s}=658 \mathrm{~nm}$ is the incident light wavelength used and $\theta$ is the scattering angle. Diffusion coefficients were obtained by fitting the data with the eq. (7). We have calculated $R_{h}$ using the value of $D$ and Stoke-Einstein relation

$D=\frac{k_{B} T}{6 \pi \eta R_{h}}$

We have used solvent viscosity $(\eta)$ to calculate $R_{h}$ as the solutions were dilute. This assumption (of using solvent viscosity) could change the absolute values of $R_{h}$. However, in the current study, rather than the absolute values of $R_{h}$, we are interested in their relative variations in different mixtures, which will not be disturbed as $R_{h}$ scales linearly with $\eta$. 


\section{Results}

\subsection{Structure}

Fig. 2 (a) and (b) shows the normalized SAXS data for the PGNP solutions and the corresponding PGNP-polymer suspensions for $\xi=0.14$ and 2.76 respectively. To visualize the structure factor and to highlight the high $q$ structure, we have multiplied the scattered intensity $(I(q))$ with the wave vector $(q)$ i.e., the normalized intensity shown in Fig. 2 is $I(q)^{*} q$. The primary peak position $\left(q^{*}\right)$ as indicated by vertical dashed lines in Fig. 2(a) and (b) is a measure of inter-particle spacing between the PGNPs, $d$, i.e. $d=2 \pi / q^{*} ; d$ for pure PGNPs was $32 \mathrm{~nm}$, which is of the order of $R_{h}$ ensuring sufficient overlap of the PGNPs and hence manifestations of the nature of polymer-PGNP interfaces i.e., the $\xi$ values can be expected in their structure/phase behavior. With addition of minimum fraction of polymers, $\mathrm{q}^{*}$ decreased along with a decay in the amplitude of primary peak (refer vertical dashed in Fig. 2(a) and (b)) for both the $\xi$ values. The shift in $\mathrm{q}^{*}$ indicates that $d$ increased with the addition of minimum fraction of polymers, while the diminishing peak amplitude implied an increase in disorder, more specifically, perturbations in core-core correlations. For the conventional colloidpolymer mixtures $[5,6]$ or star polymer-polymer mixtures $[16,50]$, it is well known that with addition of polymers, depletion induced attraction between the colloids (or star polymers) leads to aggregation of colloids and eventually to phase separation, where $\mathrm{q}^{*}$ is expected to increase. It is clear that we do not observe such effects. On the other hand, similar behavior of both the $\xi$ values is contrary to what is expected from the understanding in the melt state $[25,26,28]$. This is possibly due to the relatively high grafting

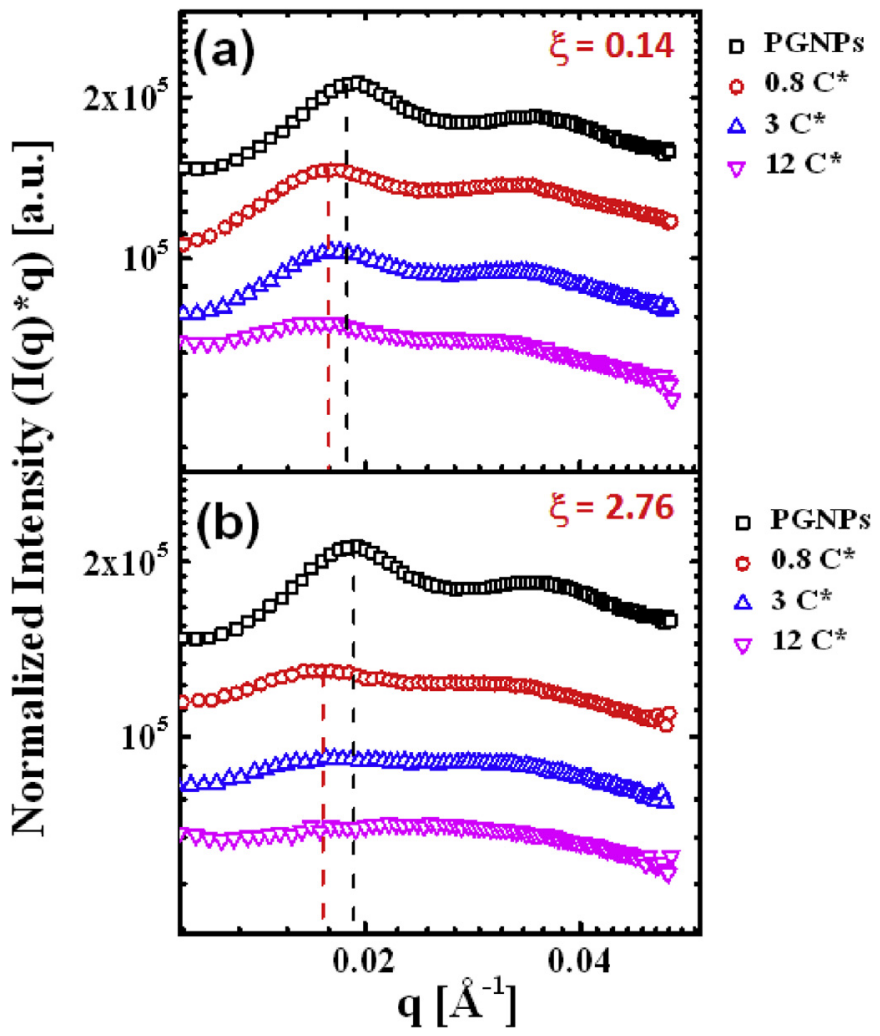

Fig. 2. Normalized intensity $\left(I(q)^{*} q\right)$ vs wave vector $q$ for the nanoparticle polymer suspensions with the $\xi$ values (a) 2.76 and (b) 0.14 is shown for highlighting the primary peak (the mean spacing between the PGNPs). Concentration of the polymers are also shown in the respective panels. The vertical dashed marks indicate the evolution of the mean spacing with the addition of polymers. density of the PGNPs used in our study. With increase in the fraction of added polymers, it can be seen that the $q^{*}$ did not decreased further. For a homogeneous dispersion of PGNPs and polymers, the $d$ should increase with increasing the fraction of added polymers and the primary peak is expected to continuously shift towards lower $q$. However, we observe that $q^{*}$ do not change with increase in the polymer concentration. It is of course possible that a weak depletion effect does exist which would lead to reduction in $d$, which is compensated by the dilution of the PGNPs due to added polymers which would lead to increase in $d$.

To summarize, we have observed an almost similar behavior for both $\xi=2.76$ and $\xi=0.14$ as far as the structural behavior is concerned. Now, let us discuss the results of microrheological studies on some of these suspensions using DWS technique.
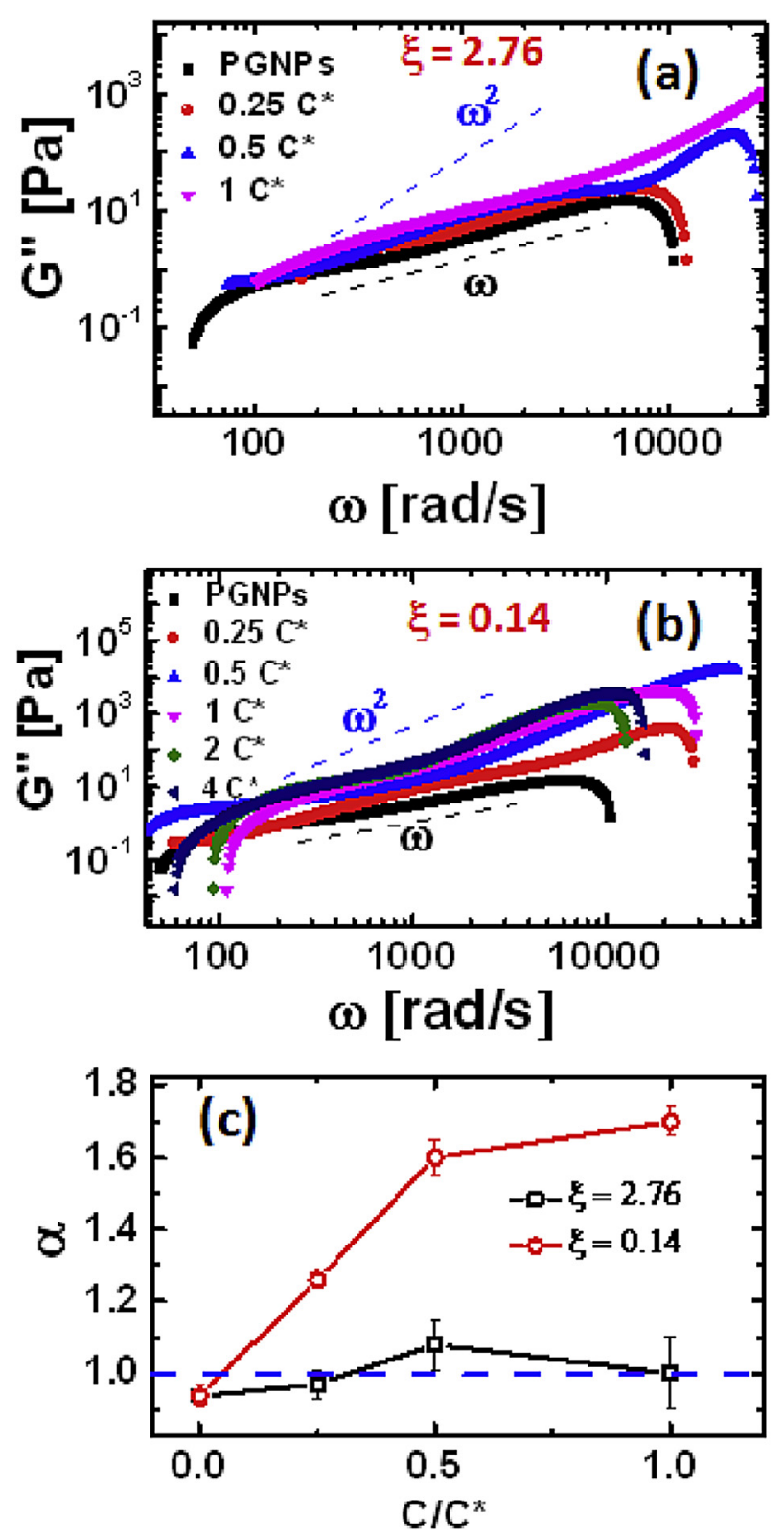

Fig. 3. Loss modulus $\left(G^{\prime \prime}\right)$ vs frequency $(\omega)$ for $\xi=2.76$ (a) and $0.14(\mathrm{~b})$ is shown. The concentrations of the polymers are indicated in the respective panels. The dashed lines in (a) and (b) indicate the nature of changes in the frequency dependence of $G^{\prime \prime}(\omega)$. (c) Exponent, $\alpha\left(G^{\prime \prime}(\omega) \sim \omega^{\alpha}\right)$ as function of the fraction of added polymers for both $\xi=2.76$ (open squares) and $\xi=0.14$ (open circles). 


\subsection{Microrheology}

In this section we discuss the macroscopic dynamics observed for the PGNP-polymer suspensions. Fig. 3(a) and (b) shows $G^{\prime \prime}(\omega)$ for mixtures with $\xi=2.76$ and 0.14 respectively. The drastic decrease in the loss modulus $\left(G^{\prime \prime}(\omega)\right)$ at the low and high frequency regions are due to the limits of the analysis and they have no physical meaning. From Fig. 3 surprising frequency dependence of $G^{\prime \prime}(\omega)$ can be noticed as a function of concentration of added polymers for $\xi=0.14$, while $\xi=2.76$ show $G^{\prime \prime}(\omega)$ similar frequency dependence for all the concentrations studied. To highlight this difference, in Fig. 3(c), we have plotted the exponent $\alpha$ in $G^{\prime \prime} \sim \omega^{\alpha}$ for both $\xi=2.76$ and $\xi=0.14$. It can be seen that while the exponent $\alpha \sim 1$ for all the concentrations studied for $\xi=2.76$ (up to $1 C^{*}$ ) it increases from $\sim 1$ to $\sim 1.6$ with increase in concentration of linear polymers for $\xi=0.14$ case (from $1 \mathrm{C}^{*}$ onwards). The increase in $\alpha$ (to values greater than one) is indicative of shear thickening of the suspensions with $\xi=0.14$, and could be driven by aggregation of PGNPs mediated by the added linear polymers or could be due to an order-disorder transition and a consequent jamming scenario [51]. Shear can be expected to enhance the probability of domain formation in these systems, eventually creating flow instabilities that could result in a steeper increase of the loss modulus with frequency. Such domain formation will not be detected in static scattering measurements but could impact the scattering dynamics. In the alternative scenario proposed by Hoffman $[52,53]$, the suspensions would have a fluid like structure, but flow instabilities could be created by the jamming of PGNPs and polymers. Thus the micro-rheology measurements clearly point to the need for additional microscopic dynamical measurements to understand the origins of such rheological transitions. The XPCS measurements on these systems sheds light on this aspect and will be discussed later. To continue further with rheological investigations, we have obtained the viscosity of the suspensions from the loss modulus, using eq. (6). Fig. 4 shows the extracted viscosity of PGNP-Polymer suspensions, as a function
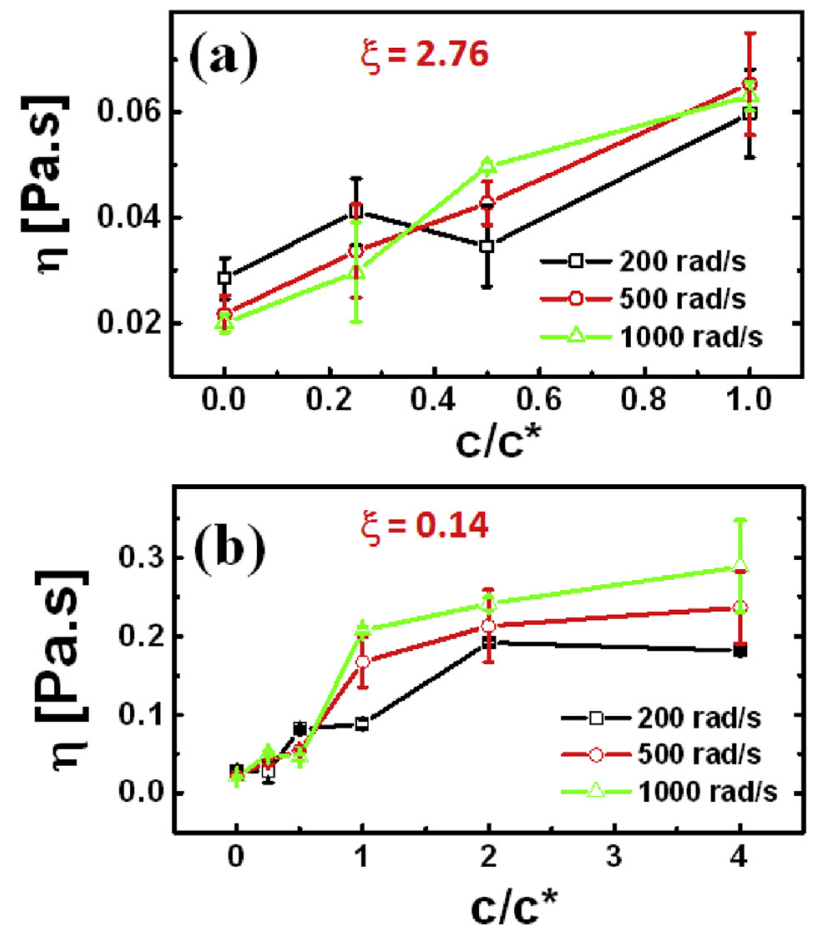

Fig. 4. Viscosity $\eta$ as a function of the concentration of added polymers $\left(C / C^{*}\right)$ at three different frequencies is shown for both (a) $\xi=2.76$ and (b) $\xi=0.14$. of the fraction of added polymers, for different frequencies. Irrespective of the $\xi$ values, it can be observed that the viscosity increases with the addition of polymers. However the variations in $\alpha$ suggests the presence of some underlying microscopic dynamical transitions which occur in the $\xi=0.14$ systems, the physics of which is not captured by DWS measurements, clearly. For this purpose, we have performed XPCS on similar PGNP-polymer suspensions.

\subsection{Dynamics}

In this section, we discuss the XPCS results on microscopic dynamics of pure PGNPs and their mixtures with linear PS in toluene. As the time windows accessible to the XPCS and DWS measurements are different, the initial concentration of the PGNPs are increased by a factor of $\sim 5$ for moving the diffusion time scales within the time window of the XPCS experiment. Fig. 5 shows the intensity auto-correlation function $\left(g_{2}(q, t)\right)$ and the corresponding intermediate scattering function (ISF, $F(q, t)$ ) of pure PGNPs for various wave vectors, $q$, as specified in the figure. The ISF of the PGNPs were fitted with an exponential function of the following
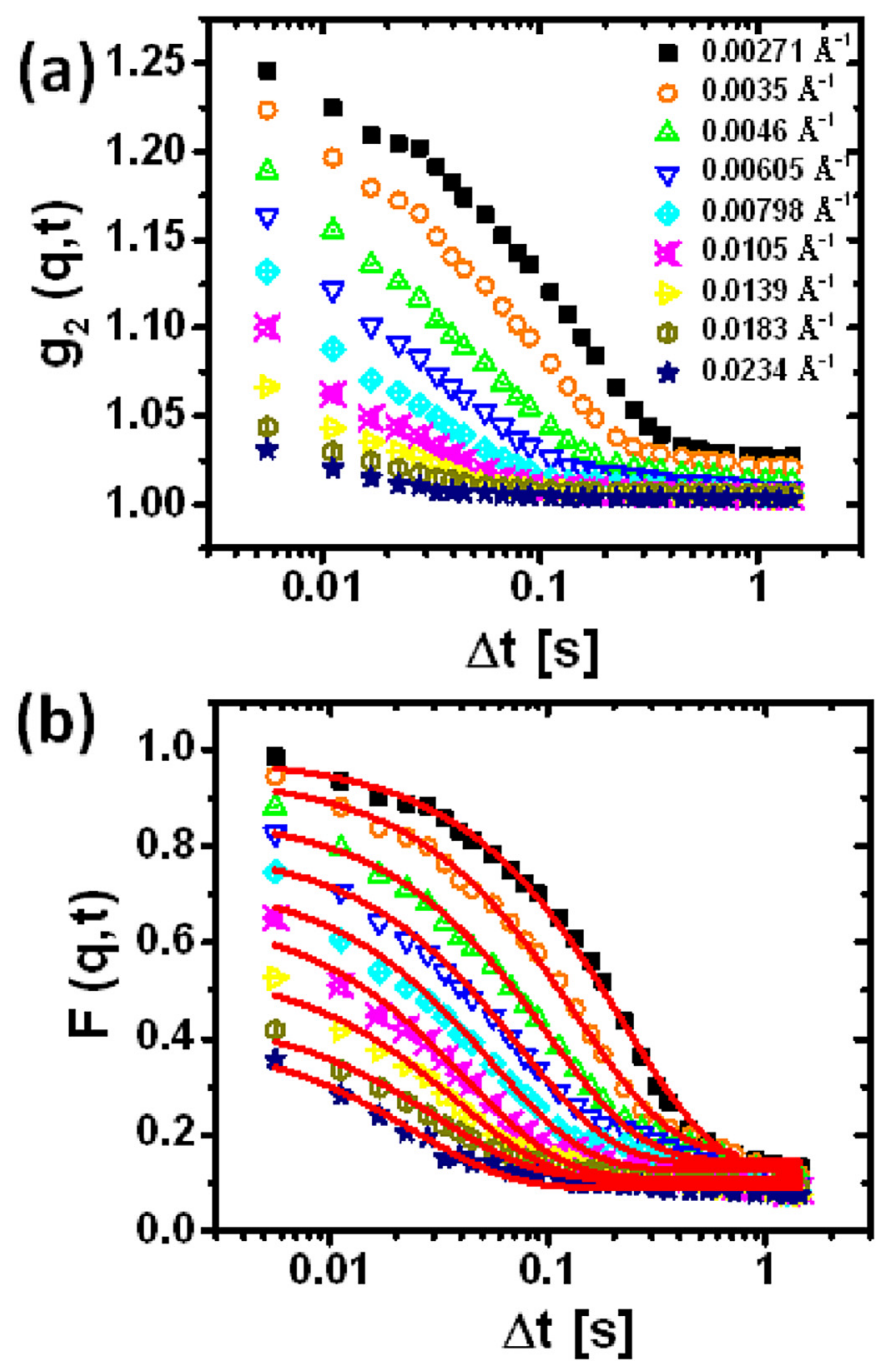

Fig. 5. (a) Two point intensity correlation function $\left(g_{2}(q, t)\right)$ and (b) the corresponding intermediate scattering function $(F(q, t))$ of pure PGNPs at various q's as indicated in the panel (a). Continuous lines in panel (b) are single exponential fits to the data (open symbols). 
form $[20]$,

$F(q, t)=A \cdot \exp \left(\frac{-t}{\tau}\right)+$ const.

where $A$ is the amplitude of the relaxation or dynamic range and $\tau$ is the relaxation time. From Fig. 5, it can be observed that the ISF of PGNPs decayed progressively faster with increase in $q$. Fig. 6 shows the ISF $\left(F_{s}(q, t)\right)$ for the PGNP-polymer suspensions. From Fig. 6, it can be noticed that for both the $\xi$ values, the dynamics of PGNPs becomes slower with the addition of polymers. Intriguingly, for mixtures with $\xi=0.14$, a transition in the nature of decay of $F_{s}(q, t)$ from exponential to logarithmic can be observed with increasing fraction of added polymers (as captured in Fig. 6(d)) with a reentrance to exponential like decay at the highest fraction of polymers studied, similar to our earlier observations [37]. In addition, the concentration after which the system relaxes logarithmically seems to match perfectly with the concentrations at which we have observed a transition in $\alpha$ from 1 to $\sim 2$ in DWS measurements. This seems to suggest a correlation between the microrheological behavior and the microscopic dynamics of the particles. On the other hand, similar to the observation of constant $\alpha$ in DWS measurements, the mixtures with $\xi=2.76$ have always decayed exponentially (refer Fig. 6(c)). These observations seem to indicate to the creation of a intrinsically different dynamic behavior for mixtures with different $\xi$ values.

To quantify the slowing down in dynamics with the addition of polymers, we extracted the relaxation times of the PGNPs in both mixtures and pristine solutions, by modeling $F_{S}(q, t)$ of the respective samples using analytical functions of the form shown in eq. (11) and below [37]:

$F_{S}(q, t)=A \cdot \exp \left(\frac{-t}{\tau}\right)+B \cdot \log \left(\frac{-t}{\tau_{l o g}}\right)+$ const.

The relaxation times ( $\tau$ from the exponential behavior) as extracted are shown in Fig. 7, for suspensions with different fraction of added polymers at representative $q$ 's, mentioned in the figure caption. It can be observed that $\tau$ increased with increase in the fraction of added polymers for both the $\xi$ values.

Now, it is sufficiently clear that the microrheology and the dynamics measurements indicate the slowing down of the dynamics with the addition of polymers. But, what is the reason behind the slower dynamics for both the systems? More importantly, what is the reason behind the transition in the nature of decay of ISF and the frequency dependence of loss modulus with addition of polymers and why the transition is observed only for mixtures with $\xi=0.14$ ?

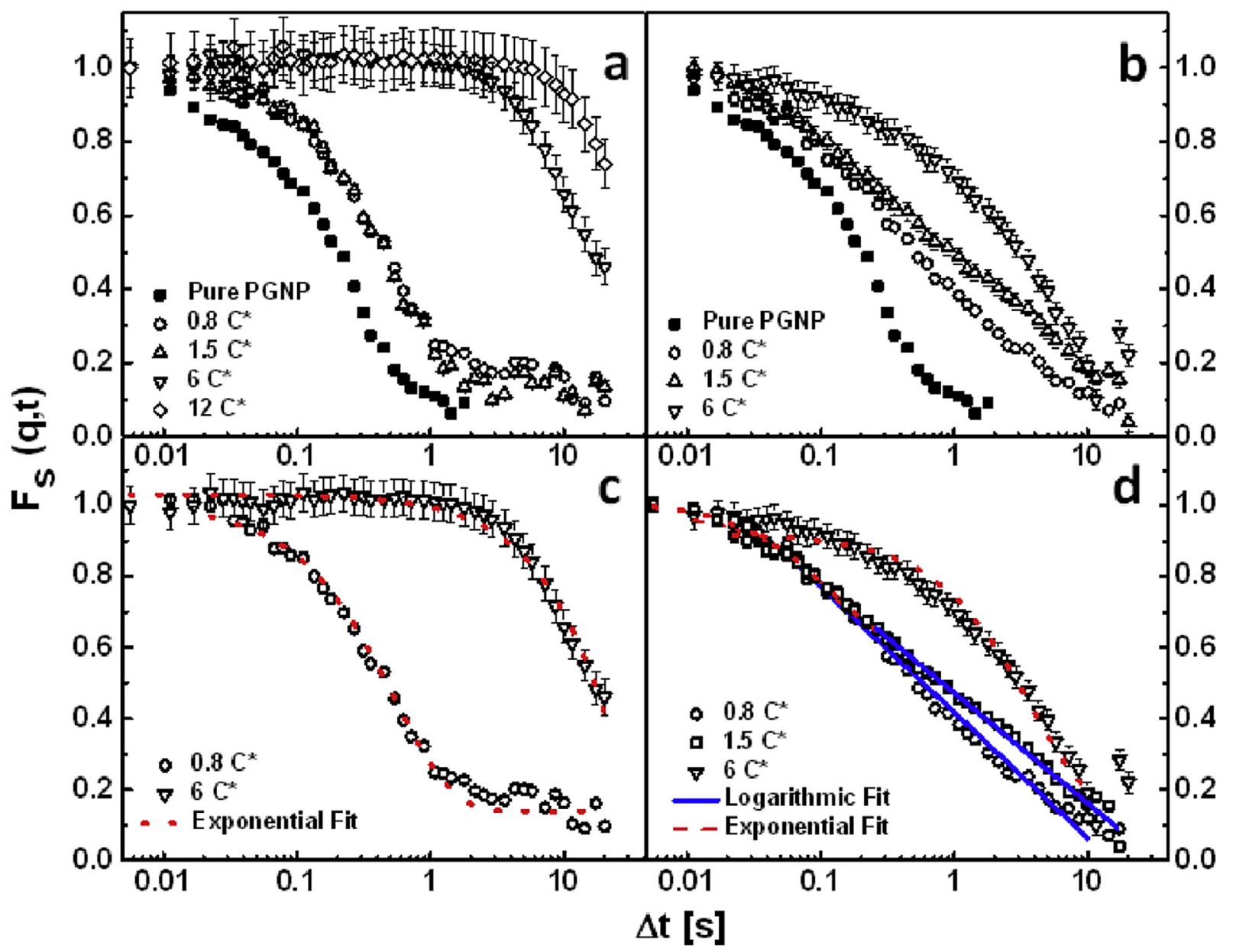

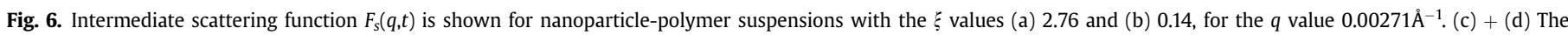

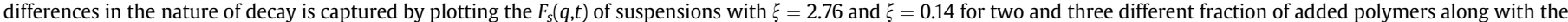

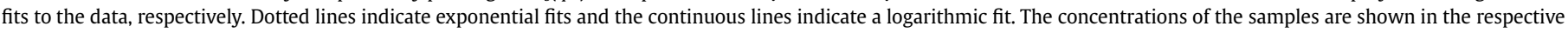
samples. At high concentrations of the added polymers, it can be observed that the nature of relaxation become exponential. 

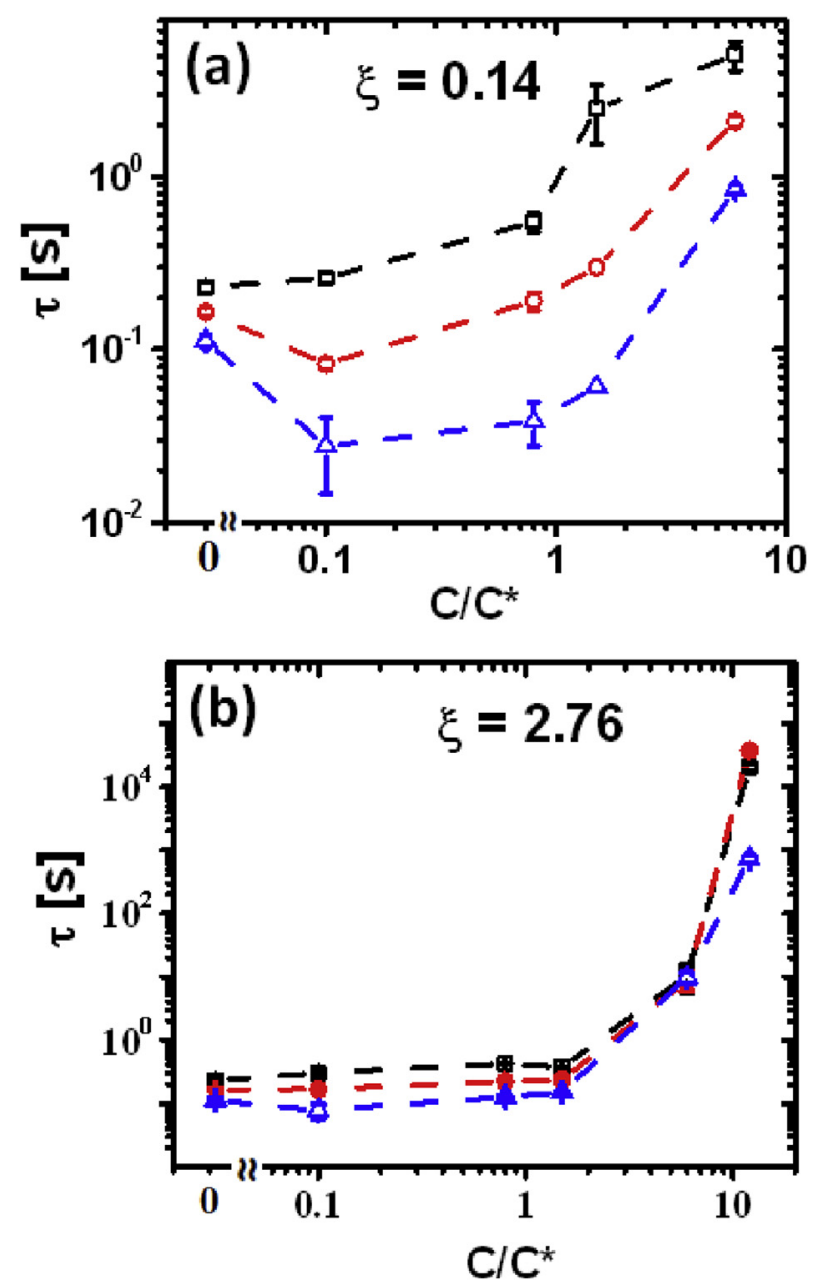

Fig. 7. $\tau$ vs $C / C^{*}$ for the suspensions with $\xi=0.14$ (a) and $\xi=2.76$ (b) is shown. Squares, circles and upward facing triangles are for $0.00271,0.0035$ and $0.0046 \AA^{-1}$ respectively.

\section{Discussions}

To address these questions, we have performed DLS experiments of the suspensions. We have performed DLS experiments, at concentrations that are reduced by a factor of $\sim 3$ in comparison with the concentrations used for DWS measurements. The size distributions obtained from DLS for both systems where compared with that of the pure PGNP suspensions and shown in Fig. 8. It can be observed that size of PGNPs increase in the presence of small polymers $(\xi=2.76)$, while the $R_{h}$ decrease in the presence of large polymers $(\xi=0.14)$. In addition, no signatures of aggregation can be observed for both the mixtures. To understand our observations, heuristically we shall begin with the PGNPs.

The hydrodynamic radius of the pure PGNPs $(\sim 30 \mathrm{~nm})$ indicate that the $R_{g}$ of the grafted polymers should follow a scaling given by $R_{g} \sim N^{\nu}$, where $3 / 5<\nu<4 / 5$ and $\mathrm{N}(=511)$ is the number of monomers constituting the grafting polymer. This indicates that the grafted polymers will be stretched and the conformation of such stretched grafted polymers will lie somewhere in between semidilute and concentrated polymer brush [54]. This stretching of the grafted chains can be expected, given the high grafting densities of the PGNPs we have used in these studies. For the mixtures with large polymers $(\xi=0.14)$, the stretching energy required to



Fig. 8. Distribution of the hydrodynamic radius $\left(R_{h}\right)$, as obtained from DLS, of pure PGNPs (open squares) is compared with the PGNPs dispersed in polymer mixtures with $\xi=2.76$ (open circles) and $\xi=0.14$ (open triangles). Solutions of $\sim 5$ wt $\%$ were used for the measurements. The dashed lines indicate the mean diameter of the PGNPs in the respective systems.

accommodate the linear polymers with in the structure formed from PGNPs will be larger than the energy gained due to interpenetration. As a consequence, the presence of $382 \mathrm{kDa}$ PS will exert an osmotic pressure on the grafted polymers resulting in the shrinkage of grafted polymers [55], which results in the decrease of the overall hydrodynamic radius of the PGNPs.

With the addition of smaller polymers $(\xi=2.76)$, we have observed almost an increase of $\sim 50 \%$ in the $R_{h}$ of the PGNPs. First of all, the magnitude of increase in $R_{h}$ rules out the possibility of aggregation of PGNPs. Since the stretching energy of the grafted polymers will be lower than the energy gained by the interpenetration of linear and grafted polymers $[25,26,28]$, the polymers in the mixtures with $\xi=2.76$ can be expected to interpenetrate with the grafted polymers. As a consequence of interpenetration, the grafted polymer will stretch further to acquire a concentrated brush conformation $(\nu=4 / 5)$ [54]. However, due to the high grafting density of our PGNPs a complete interpenetration will not be possible. As a consequence, a scenario of partially interpenetrated linear chains around the PGNPs can be expected behind the increase in the $R_{h}$ of PGNPs in the presence of polymers with $\xi=2.76$. Refer Fig. 9 for the proposed model of the morphology mixtures in comparison with the pure PGNPs. In short, as shown in Fig. 9, the addition of large polymers breaks the network of the PGNPs, while the addition of small polymers stabilizes the PGNP network by enhanced interpenetration.

Now let us turn our attention to the observations of rheology and dynamics measurements. Since the XPCS and DWS experiments, were performed at higher concentrations the changes observed from DLS would be enhanced further. For the mixtures with $\xi=0.14$, the addition of minimum fraction of polymers will break/melt the structure (indicated by the increase in the interparticle separation shown in Fig. 2) formed by PGNPs due to the osmotic shrinkage of the grafted polymers [55]. As a result, a decrease in the relaxation time of PGNPs has been observed (refer Fig. 7(a)). With further increase in the fraction of added polymers, due to the possible over crowding of polymers, the viscosity (and the relaxation time) increases slightly as observed in Figs. 4 and 7. 


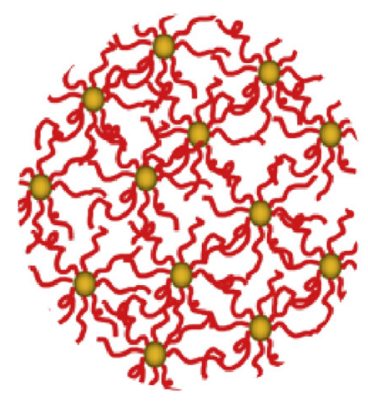

Pure PGNPs

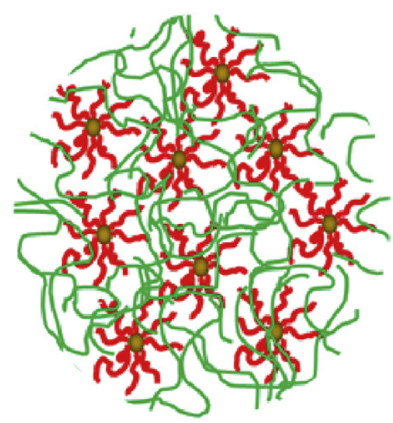

$\xi=0.14$

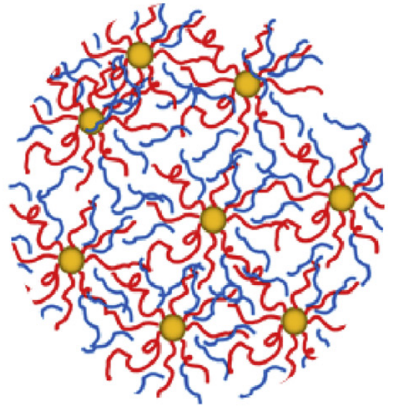

$\xi=\mathbf{2 . 7 6}$

Fig. 9. Schematic of the proposed morphology of the PGNPs and polymers for both the mixtures are shown in comparison with the morphology of pure PGNPs.

Due to high fraction of linear polymers, the grafted layer of the PGNPs will further shrink leading to a disordered and frustrated state (refer Fig. 9) approaching stability through long time logarithmic relaxations. This also suggests that the shear thickening behavior, observed in our DWS measurements, is likely due to a concentration dependent order-disorder transition in the suspensions with $\xi=0.14$. Simple domain formation or aggregation would have simply led to a simple exponential ISF with possibly higher characteristic relaxation time. The fact that we observe long time logarithmic relaxations indicates the tendency of the PGNPs towards occupying a low energy configuration as is the case in concentrated aging colloidal suspensions [56]. Curiously, logarithmic relaxations have been predicted and observed for single or two component mixtures of conventional colloids and star polymers that are close to higher order dynamic singularities $[5,6,14-17,50,57-59]$. These singularities, in star polymers is observed due to the low size asymmetry (size ratios of the two components is closer to 1) as is the case for our mixtures with $\xi=0.14(\lambda \approx 1$ refer Table 2$)$. It is interesting to note that the behavior of PGNPs, especially in solution and for such large grafting density and grafted chain molecular weight, is likely to be very close to that of star polymers as has been suggested earlier $[19,21,37,60,61]$. In the binary mixtures of star polymers for $\lambda \approx 2$, a transition from asymmetric to double glass type is observed (in simulations) through logarithmically decaying correlators. We believe that the situation of our system with $\xi=0.14$ will be similar. At the highest concentration of added polymers $\left(6 C^{*}\right)$ we have observed a re-entrance in ISF from logarithmic to exponential (refer Fig. 6) similar to our earlier observation [37]. This is, however, different from the theoretical predictions of [17] although for star polymers very high concentration (of additives) has not been experimentally realized. It is important to note that the volume fraction of the PGNPs used in the experiments were much smaller compared to that of a glassy phase, as considered in Ref. [17]. This fact coupled with the high concentration of added linear polymers, used in our experiments, can possibly explain the re-entrance behavior. Further, the observed dynamical transition could be indicative, more of a liquid-liquid transition, which might be treated as precursor to the corresponding glass-glass transition, at higher PGNP volume fractions, as predicted by Ref. [17]. According to the theory of Likos et al., we can also anticipate such dynamics for the linear polymers. However, in the presence of solvent and the gold core, scattering from the linear polymers will be very weak and negligible and hence will not contribute significantly in the intermediate scattering function such that possible signatures of similar dynamical transitions in the linear polymer component cannot be detected. On the other hand, for the mixtures with $\xi=2.76$, the interpenetration between the linear and grafted polymers would further increase with increasing the fraction of added polymers. As a consequence the distance between the PGNPS should further increase, as shown in our SAXS data by the peaks shifting towards lower $q$. Further, as discussed earlier (and shown in Fig. 9), due to the partial interpenetration there is a possibility of layer of linear polymers carried along with the PGNPs. This will lead to increase in the viscosity (or relaxation time) as observed from our DWS and XPCS measurements. Hence, the increase in relaxation time with increasing concentration of added polymers in $\xi=2.76$ can be viewed as the diffusion of particles with increasing size (due to the swollen brush and to the possibility of a layer of linear polymers around the PGNPs). Additional effects due to the rather high concentrations for the overlap of linear polymers will also reflect in the large increase in the relaxation time, as observe from XPCS measurements. Thus, for systems with $\xi=2.76$, the relaxation mechanism is a simple random diffusion of PGNPs (and a surrounding partially interpenetrated layer of linear polymers) in high viscous medium which results in simple exponential decay of the ISF.

\section{Conclusion}

To conclude, we have observed an experimental signature for the osmotic shrinkage (for mixtures with $\xi=0.14$ ) and the swelling of the brush (for $\xi=2.76$ ), along with the associated changes in the conformations of the grafted polymers. In contrary to the expectation from the conventional colloids and star polymers, we did not observed the presence of any depletion interactions in our suspensions. DWS microrheology measurements reveal surprising transition which is echoed by a corresponding dynamical transition in XPCS. We have proposed a (morphological) model to explain the observed dynamical differences with the addition of polymers. Our work clearly indicates the need for further experimental and theoretical investigations for the proper understanding of PGNPpolymer suspensions with possible implication for binary mixtures of related soft colloidal systems.

\section{Acknowledgements}

Authors thank the Department of Science and Technology (SERB) and Nanomission, India for the financial support and Saha Institute of Nuclear Physics, India for facilitating the experiments at the PETRA-III, Hamburg, Germany. The authors would also like to acknowledge Institute Nanoscience Initiative (INI), AFMM,IISc for providing access to TEM facilities. 


\section{References}

[1] D.A.S.W.B. Russel, W.R. Schowalter, Colloidal Dispersions, Cambridge Monographs on Mechanics, 1989, http://dx.doi.org/10.1017/CBO9780511608810 (ISBN: 978-0521426008).

[2] H.N.W. Lekkerkerker, R. Tuinier, Colloids and the Depletion Interaction, in: Lecture Notes in Physics, 833, 2011, http://dx.doi.org/10.1007/978-94-0071223-2 (ISBN: 978-94-007-1222-5).

[3] P.J. Lu, D.A. Weitz, Colloidal particles: crystals, glasses, and gels, Annu. Rev. Condens. Matter Phys. 4 (1) (2013) 217-233, http://dx.doi.org/10.1146 annurev-conmatphys-030212-184213.

[4] Y.M. Joshi, Dynamics of colloidal glasses and gels, Annu. Rev. Chem. Biomol Eng. 5 (1) (2014) 181-202, http://dx.doi.org/10.1146/annurev-chembioeng060713-040230.

[5] K.N. Pham, A.M. Puertas, J. Bergenholtz, S.U. Egelhaaf, A. Moussaïd, P.N. Pusey, A.B. Schofield, M.E. Cates, M. Fuchs, W.C.K. Poon, Multiple glassy states in simple model system, Science 296 (2002) 104-106, http://dx.doi.org/10.1126 science.1068238.

[6] T. Eckert, E. Bartsch, Re-entrant glass transition in a colloid-polymer mixture with depletion attractions, Phys. Rev. Lett. 89 (2002) 125701, http:// dx.doi.org/10.1103/PhysRevLett.89.125701.

[7] A.N. Semenov, Theory of colloid stabilization in semidilute polymer solutions, \begin{tabular}{lllll}
\hline Macromolecules 41 & 41 & (2008) & $2243-2249$, & http://dx.doi.org/10.1021
\end{tabular} ma702536c.

[8] S. Asakura, F. Oosawa, Interaction between particles suspended in solutions of macromolecules, J. Polym. Sci. 33 (126) (1958) 183-192, http://dx.doi.org/ 10.1002/pol.1958.1203312618.

[9] J. Sprakel, J. van der Gucht, M.A. Cohen Stuart, N.A.M. Besseling, Rouse dy namics of colloids bound to polymer networks, Phys. Rev. Lett. 99 (2007) 208301, http://dx.doi.org/10.1103/PhysRevLett.99.208301.

[10] D. Vlassopoulos, M. Cloitre, Tunable rheology of dense soft deformable colloids, Curr. Opin. Colloid Interface Sci. 19 (6) (2014) 561-574, http: dx.doi.org/10.1016/i.cocis.2014.09.007.

[11] P. Akcora, H. Liu, S. K. Kumar, J. Moll, NewAuthor5, B. C. Benicewicz, L. S Schadler, D. Acehan, A. Z. Panagiotopoulos, V. Pryamitsyn, V. Ganesan, J. Ilavsky, P. Thiyagarajan, R. H. Colby, J. F. Douglas, Anisotropic self-assembly of spherical polymer-grafted nanoparticles, Nat. Mat., 8. doi:http://dx.doi.org/10. 1038/NMAT2404.

[12] Z. Zhang, N. Xu, D.T.N. Chen, P. Yunker, A.M. Alsayed, K.B. Aptowicz, P. Habdas A.J. Liu, S.R. Nagel, A.G. Yodh, Thermal vestige of the zero-temperature jamming transition, Nature 459 (7244) (2009) 230-233, http://dx.doi.org 10.1038/nature07998.

[13] J. Mattsson, H.M. Wyss, A. Fernandez-Nieves, K. Miyazaki, Z. Hu, D.R. Reichman, D.A. Weitz, Soft colloids make strong glasses, Nature 462 (7269) (2009) 83-86, http://dx.doi.org/10.1038/nature08457.

[14] C.N. Likos, Soft matter with soft particles, Soft Matter 2 (6) (2006) 478-498 http://dx.doi.org/10.1039/b601916c.

[15] C. Mayer, E. Zaccarelli, E. Stiakakis, C.N. Likos, F. Sciortino, A. Munam, M. Gauthier, N. Hadjichristidis, H. Iatrou, P. Tartaglia, H. Lowen, D. Vlassopoulos, Asymmetric caging in soft colloidal mixtures, Nat. Mater. 7 (10) (2008) 780-784, http://dx.doi.org/10.1038/nmat2286.

[16] E. Stiakakis, D. Vlassopoulos, C.N. Likos, J. Roovers, G. Meier, Polymer-mediated melting in ultrasoft colloidal gels, Phys. Rev. Lett. 89 (20) (2002) 208302 http://dx.doi.org/10.1103/PhysRevLett.89.208302.

[17] C. Mayer, F. Sciortino, C.N. Likos, P. Tartaglia, H. Löwen, E. Zaccarelli, Multiple glass transitions in star polymer mixtures: insights from theory and simulations, Macromolecules 42 (1) (2009) 423-434, http://dx.doi.org/10.1021/ ma801894x.

[18] D. Meng, S.K. Kumar, J.M.D. Lane, G.S. Grest, Effective interactions between grafted nanoparticles in a polymer matrix, Soft Matter 8 (2012) 5002, http:/ dx.doi.org/10.1039/c2sm07395a.

[19] S. Srivastava, S. Chandran, A. K. Kandar, C. K. Sarika, J. K. Basu, S. Narayanan, A Sandy, Communication: unusual dynamics of hybrid nanoparticles and their binary mixtures, J. Chem. Phys., 133, 15. doi:http://dx.doi.org/10.1063/1. 3495480.

[20] S. Chandran, C. K. Sarika, A. K. Kandar, J. K. Basu, S. Narayanan, A. Sandy, Reentrant behavior in dynamics of binary mixtures of soft hybrid nanocolloids and homopolymers, J. Chem. Phys., 135 (13). http://dx.doi.org/10.1063/1. 3644930.

[21] S. Chandran, S. Saw, A. K. Kandar, C. Dasgupta, M. Sprung, J. K. Basu, Suspensions of polymer-grafted nanoparticles with added polymers structure and effective pair-interactions, J. Chem. Phys., 143 (8). http://dx.doi.org/10 1063/1.4929438.

[22] S. C. Warren, L. C. Messina, L. S. Slaughter, M. Kamperman, O. Zhou, S. M Gruner, F. J. DiSalvo, U. Wiesner, Ordered mesoporous materials from metal nanoparticle-block copolymer self-assembly, Science, 320. doi:http://dx.doi org/10.1126/science.1159950.

[23] R. B. Thompson, V. V. Ginzburg, M. W. Matsen, A. C. Balazs, Predicting the mesophases of copolymer-nanoparticle composites, Science, 292. doi:http:/ dx.doi.org/10.1126/science.1060585.

[24] S. Chandran, N. Begam, V. Padmanabhan, J.K. Basu, Confinement enhances dispersion in nanoparticle-polymer blend films, Nat. Commun. 5 (2014) 3697, http://dx.doi.org/10.1038/ncomms4697.

[25] S.K. Kumar, N. Jouault, B. Benicewicz, T. Neely, Nanocomposites with polymer grafted nanoparticles, Macromolecules 46 (2013) 3199-3214, http:// dx.doi.org/10.1021/ma4001385.

[26] P.F. Green, The structure of chain end-grafted nanoparticle/homopolymer nanocomposites, Soft Matter 7 (2011) 7914, http://dx.doi.org/10.1039/ c1sm05076a.

[27] D.F. Sunday, D.L. Green, Thermal and rheological behavior of polymer grafted nanoparticles, Macromolecules 48 (2015) 8651-8659, http://dx.doi.org/ 10.1021/acs.macromol.5b00987.

[28] S. Chandran, N. Begam, J. K. Basu, Dispersion of polymer grafted nanoparticles in polymer nanocomposite films: insights from surface X-ray scattering and microscopy, J. Appl. Phys., 116 (22). http://dx.doi.org/10.1063/1.4902964.

[29] V. Ganesan, C.J. Ellison, V. Pryamitsyn, Mean-field models of structure and dispersion of polymer-nanoparticle mixtures, Soft Matter 6 (2010) 4010, http://dx.doi.org/10.1039/b926992d.

[30] A. Jayaraman, Polymer grafted nanoparticles: effect of chemical and physical heterogeneity in polymer grafts on particle assembly and dispersion, J. Polym. Sci. B Polym. Phys. 51 (2013) 524-534, http://dx.doi.org/10.1002/polb.23260.

[31] C. Chevigny, F. Dalmas, E. di Cola, D. Gigmes, D. Bertin, F. Boué, J. Jestin, Polymer-grafted-nanoparticles nanocomposites: dispersion, grafted chain conformation, and rheological behavior, Macromolecules 44 (2011) 122-133. http://dx.doi.org/10.1021/ma101332s.

[32] C. Durand-Gasselin, N. Sanson, N. Lequeux, Reversible controlled assembly of thermosensitive polymer-coated gold nanoparticles, Langmuir 27 (20) (2011) 12329-12335, http://dx.doi.org/10.1021/la2023852.

[33] B. Natarajan, T. Neely, A. Rungta, B.C. Benicewicz, L.S. Schadler, Thermomechanical properties of bimodal brush modified nanoparticle composites, Macromolecules 46 (2013) 4909-4918, http://dx.doi.org/10.1021/ ma400553c.

[34] Y. Li, P. Tao, A. Viswanath, B.C. Benicewicz, L.S. Schadler, Bimodal surface ligand engineering: the key to tunable nanocomposites, Langmuir 29 (4) (2013) 1211-1220, http://dx.doi.org/10.1021/la3036192.

[35] B. Palli, V. Padmanabhan, Chain flexibility for tuning effective interactions in blends of polymers and polymer-grafted nanoparticles, Soft Matter $10(2014)$ 6777, http://dx.doi.org/10.1039/C4SM00991F.

[36] H. Feng, M. Bhmer, R. Fokkink, J. Sprakel, F. Leermakers, Reentrant stabilization of grafted nanoparticles in polymer solutions, J. Phys. Chem. B 119 (40) (2015) 12938-12946, http://dx.doi.org/10.1021/acs.jpcb.5b05504.

[37] A.K. Kandar, I.K. Basu, S. Narayanan, A. Sandy, Anomalous structural and dynamical phase transitions of soft colloidal binary mixtures, Soft Matter 8 (2012) 10055-10060, http://dx.doi.org/10.1039/C2SM25774B.

[38] S. Srivastava, J.H. Shin, L.A. Archer, Structure and rheology of nanoparticlepolymer suspensions, Soft Matter 8 (2012) 4097, http://dx.doi.org/10.1039/ c2sm06889c

[39] N. Begam, S. Chandran, M. Sprung, J.K. Basu, Anomalous viscosity reduction and hydrodynamic interactions of polymeric nanocolloids in polymers, Macromolecules 48 (2015) 6646-6651, http://dx.doi.org/10.1021/ acs.macromol.5b00759.

[40] M.K. Corbierre, N.S. Cameron, M. Sutton, S.J. Mochrie, L.B. Lurio, A. Rühm, R.B. Lennox, Polymer-stabilized gold nanoparticles and their incorporation into polymer matrices, I. Am. Chem. Soc. 123 (42) (2001) 10411-10412, http://dx.doi.org/10.1021/ja0166287.

[41] P. Kraft, A. Bergamaschi, C. Bronnimann, R. Dinapoli, E.F. Eikenberry, H. Graafsma, B. Henrich, I. Johnson, M. Kobas, A. Mozzanica, C.M. Schleputz, B. Schmitt, Characterization and calibration of PILATUS detectors, IEEE Trans. Nucl. Sci. 56 (2009) 758-764, http://dx.doi.org/10.1109/TNS.2008.2009448.

[42] C. Ponchut, J.M. Rigal, J. Clément, E. Papillon, A. Homs, S. Petitdemange, MAXIPIX, a fast readout photon-counting X-ray area detector for synchrotron applications, J. Instrum. 6 (2011) C01069, http://dx.doi.org/10.1088/17480221/6/01/C01069.

[43] J. Möller, Y. Chushkin, S. Prevost, T. Narayanan, Multi-speckle X-ray photon correlation spectroscopy in the ultra-small-angle X-ray scattering range, J. Synchrotron Radiat., 23, 4. doi:http://dx.doi.org/10.1107/ S1600577516008092.

[44] Z. Evenson, B. Ruta, S. Hechler, M. Stolpe, E. Pineda, I. Gallino, R. Busch, X-ray photon correlation spectroscopy reveals intermittent aging dynamics in a metallic glass, Phys. Rev. Lett. 115 (2015) 175701, http://dx.doi.org/10.1103/ PhysRevLett.115.175701.

[45] D.A. Weitz, I.X. Zhu, D.J. Durian, H. Gang, D.J. Pine, Diffusing-wave spectroscopy: the technique and some applications, Phys. Scr. 1993 (T49B) (1993) 610. http://stacks.iop.org/1402-4896/1993/i=T49B/a=040.

[46] T.G. Mason, D.A. Weitz, Optical measurements of frequency-dependent linear viscoelastic moduli of complex fluids, Phys. Rev. Lett. 74 (1995) 1250-1253, http://dx.doi.org/10.1103/PhysRevLett.74.1250.

[47] B.R. Dasgupta, S.-Y. Tee, J.C. Crocker, B.J. Frisken, D.A. Weitz, Microrheology of polyethylene oxide using diffusing wave spectroscopy and single scattering, Phys. Rev. E 65 (2002) 051505, http://dx.doi.org/10.1103/PhysRevE.65.051505. http://link.aps.org/doi/10.1103/PhysRevE.65.051505.

[48] R. Larson, The Structure and Rheology of Complex Fluids, Topics in Chemical Engineering, OUP USA, 1999. https://books.google.de/books?id=Vt9fw/_ pf1LUC.

[49] B.J. Berne, R. Pecora, Dynamic Light Scattering: with Applications to Chemistry, Biology, and Physics, Courier Corporation, 1976.

[50] M. Camargo, S.A. Egorov, C.N. Likos, Cluster formation in star-linear polymer mixtures: equilibrium and dynamical properties, Soft Matter 8 (2012) 4177, http://dx.doi.org/10.1039/c2sm06849d. 
[51] R.L. Hoffman, Explanations for the cause of shear thickening in concentrated colloidal suspensions, J. Rheol. 42 (1) (1998) 111-123, http://dx.doi.org 10.1122/1.550884.

[52] R.L. Hoffman, Discontinuous and dilatant viscosity behavior in concentrated suspensions. i. observation of a flow instability, Trans. Soc. Rheol. 16 (1) (1972) 155-173, http://dx.doi.org/10.1122/1.549250. http://scitation.aip.org/ content/sor/journal/tsor/16/1/10.1122/1.549250.

[53] R. Hoffman, Discontinuous and dilatant viscosity behavior in concentrated suspensions. ii. theory and experimental tests, J. Colloid Interface Sci. 46 (3) (1974) 491-506, http://dx.doi.org/10.1016/0021-9797(74)90059-9. http:// www.sciencedirect.com/science/article/pii/0021979774900599.

[54] D. Dukes, Y. Li, S. Lewis, B. Benicewicz, L. Schadler, S.K. Kumar, Conformational transitions of spherical polymer brushes: synthesis, characterization, and theory, Macromolecules 43 (2010) 1564-1570, http://dx.doi.org/10.1021/ ma901228t.

[55] A. Wilk, S. Huißmann, E. Stiakakis, J. Kohlbrecher, D. Vlassopoulos, C.N. Likos, G. Meier, J.K.G. Dhont, G. Petekidis, R. Vavrin, Osmotic shrinkage in star/linear polymer mixtures, Eur. Phys. J. E 32 (2) (2010) 127-134, http://dx.doi.org/ 10.1140/epje/i2010-10607-2.
[56] L. Cipelletti, L. Ramos, Slow dynamics in glassy soft matter, J. Phys. Condens Matter 17 (6) (2005) R253. http://stacks.iop.org/0953-8984/17/i=6/a=R01.

[57] X. Lu, S.G.J. Mochrie, S. Narayanan, A.R. Sandy, M. Sprung, How a liquid becomes a glass both on cooling and on heating, Phys. Rev. Lett. 100 (2008) 045701, http://dx.doi.org/10.1103/PhysRevLett.100.045701.

[58] K.N. Pham, S.U. Egelhaaf, P.N. Pusey, W.C.K. Poon, Glasses in hard spheres with short-range attraction, Phys. Rev. E 69 (2004) 011503, http://dx.doi.org 10.1103/PhysRevE.69.011503.

[59] S.-H. Chen, W.-R. Chen, F. Mallamace, The glass-to-glass transition and its end point in a copolymer micellar system, Science 300 (2003) 619-622, http:// dx.doi.org/10.1126/science.1082364.

[60] G.H. Fredrickson, A.J. Liu, F.S. Bates, Entropic corrections to the flory-huggins theory of polymer blends: architectural and conformational effects, Macromolecules 27 (9) (1994) 2503-2511, http://dx.doi.org/10.1021/ma00087a019 arXiv:http://dx.doi.org/10.1021/ma00087a019.

[61] G.D. Smith, D. Bedrov, Dispersing nanoparticles in a polymer matrix: are long dense polymer tethers really necessary? Langmuir 25 (19) (2009) 11239-11243, http://dx.doi.org/10.1021/la902329v pMID: 19788206. arXiv: http://dx.doi.org/10.1021/la902329v. 